\title{
Quality of life among elderly population
}

Ioanna V. Papathanasiou ${ }^{1}$, Anna Rammogianni ${ }^{1}$, Dimitrios Papagiannis ${ }^{1}$, Konstantinos Tsaras $^{1}$, Foteini Kaberi ${ }^{1}$, Lamprini Kontopoulou ${ }^{1}$, Anna Neroliatsiou ${ }^{2}$, Evangelos C. Fradelos ${ }^{1}$

${ }^{1}$ University of Thessaly, Larissa, Greece

${ }^{2}$ University Hospital of Larissa, Greece

\section{International Conference "Mental Health: global challenges of XXI century" Conference Abstract}

Introduction: Quality of Life (QoL) among elderly is an important issue that reflects the status of well-being of this vulnerable population.

Aim: This study aims to assess quality of life among elderly population and to examine possible correlations with associated demographic, social and health factors.

Material and Method: A cross sectional study was conducted, in which 257 elderlies from Greece were participated. For the data collection the WHOQOL-BREF (30-items Greek version) questionnaire was used as well as a questionnaire with questions about demographic data, social \& health factors. Descriptive statistics such as frequencies, means, percentages and standard deviations have been utilized. Inferential statistics such as t-test and pearson $r$ correlation have been used to determined correlations between relevant variables. Level of significance accepted is $\mathrm{p}<0.05$.

Results: From the total 257 elderlies 55.6\% $(n=143)$ were women and $44.4 \%(n=114)$ were men with a mean age $75.12 \pm 8.39$. The mean score of overall QoL is $14.14 \pm 2.87$ and the mean of each factor of WHOQOL-BREF is $13.56 \pm 2.79$ for physical health, $13.61 \pm 2.74$ for mental health, $13.72 \pm 2.60$ for social relationships and $13.70 \pm 1.96$ for environment. Age, marital status, number of children, level of education, residence area, lifestyle, chronic diseases and serious illnesses are the factors that affects levels of QoL among Greek elderly population

Conclusions: Results indicates that levels of QoL between elderly are moderate and many demographic, social and health factors are correlated with QoL status.

\section{References}

Abdala, G. A., Kimura, M., Oliveira Duarte, Y. A., Lebrão, M. L. \& Santos, B. (2015). Religiousness and health-related quality of life of older adults. Revista de Saúde Pública, 49(1):49-55.

Borglin, G., Edberg, A.-K., \& Rahm Hallberg, I. (2005). The experience of quality of life among older people. Journal of Aging Studies, 19(2):201-220.

Bowling, A., Banister, D., Sutton, S., Evans, O. \& Windsor, J. (2002). A multidimensional model of the quality of life in older age. Aging \& Mental Health, 6(4): 355-371.

Ginieri-Coccossis M, Triantafillou E, Tomaras V, Soldatos C, Mavreas V, Christodoulou G. (2012). Psychometric properties of WHOQOL-BREF in clinical and healthy Greek populations: Incorporating new culture- relevant items. Psychiatriki 2012, 23(2):130-42.

Wood, N. F., LaCroix, A. Z., Gray, S. L., Aragaki, A., Cochrane, B. B., Brunner, R. L., Masaki, K., Murray, A. \& Newman, A. B. (2005). Frailty: Emergence and Consequences in Women Aged 65 and Older in the Women's Health Initiative Observational Study. Journal of the American Geriatrics Society, 53(8):1321-1330

Keywords: Elderly, WHOQOL-BREF, Quality of Life 